\title{
Synthetic considerations on the origin and emergence of human language
}

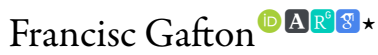 \\ Faculty of Letters, "Alexandru Ioan Cuza" University, Bd. Carol I 11, 700506 Iași, Romania
}

\section{Article info}

History:

Received October 20, 2021

Accepted October 26, 2021

Published December 12, 2021

Key words:

the origin of language

evolution

biosocial behaviour

multimodal

\begin{abstract}
Human (vocal-articulated) language may be regarded both as a structure and organ, and as an instrument and biosocial behaviour. Its foundation is material and motor, and its development procedural-historical. Examining the relationship between the initial moment (gesture) and the actual result (vocalarticulated language) - at the motor and neural levels—reveals that mental activities occur and develop as a result of the biological organism's interactions with its natural and social environment. Therefore, language appears not as a consequence of a revolution or of an evolutionary jump, but as a result of organic and gradual developments occurring in a biosocial environment and having the same kind of premises. The many structures contributing to its appearance (osseous, muscular, nervous, genetic) were directed by social and biological events generating behaviours whose exercise led to certain developments.

Having achieved structural and behavioral diversification, and in so far as it could benefit evolution, such a development was exploited by the biological and social organism, such that-irrespective of space, time, or the aspects of a particular context-above a certain level of evolution, the various human communities spread throughout the globe exhibited the intrinsic tendency of developing this ability.
\end{abstract}

"Prima sum: primatum nil a me alienum puto."

(Earnest Albert Hooton)

Rejection and denial. After generations of scholars have struggled to try to figure out the genesis of speech and of language, linguists - focusing on considerations outside of science-decided to eliminate from their searches the question of genesis and the emergence of speech and of language, reasoning that it is not in the field of linguistics and, in fact, does not interest scientific research, being insurmountable and useless.

In this way they rejected the natural path of scientific research, permeated by the truth that, in order to really understand an entity or a process, they must be studied in the aspects of genesis and appearance, practice and development, functioning, adaptation and evolution of that reality. This has affected organically: a) the exhaustive, profound and, ultimately, real character of knowledge; b) the ability of linguistics to truly understand speech and language, to enhance (its) knowledge; c) the relationship of linguistics with the sciences that appeal to and build with reliable knowledge.

On the other hand, because all the entities and processes that the sciences deal with are investigated with maximum dedication under the mentioned aspects, the attitude of linguistics provoked its own isolation, since renouncing the preoccupation with the question of origin, linguistics could no longer present itself in front of the upstream sciences with data that could be incorporated into the material interwove through the collective contribution of the sciences, but, at most, with data that remained next to them, as they spoke only about and for themselves.

^Email address algafton@gmail.com. 
Even so, things would have remained the same, since the concrete needs of linguistics seemed to be met by the kind of fragmentary and superficial knowledge that it had adopted and that characterized it. A few decades ago, however, research from other sciences reached the area where-in order to progress in scale and depth, but also to be able to use with confidence some of the data whose research was the responsibility of linguistics - needed to clarify the question of genesis and the emergence of speech and of language. Those sciences continued to remain negligible or not at all interested in the research, results and conclusions that linguistics offered-which is quite natural because, on the one hand, many of the data that linguistics can procure serve only to show that the entire exercise can generate something, on the other hand, because the results obtained without taking into account the genesis and appearance can only be incomplete (at best) or erroneous (usually) -, their interest focusing on the origin and constitutive processes of speech and of language. That is why the resetting of the entire process was also initiated by researchers belonging to other fields ${ }^{1}$.

Acceptance and search. At first, the attempts to find out the factors and causes of the emergence of human language went down unidirectional paths, but gradually it was understood that human language does not appear as a consequence of a single factor or of a biological or/and social occurrence, but is the result of the action of a bundle of factors and events, some developed from others, all interwoven at a certain moment and having deep and concrescued roots-obvious or latent. That is, an evolution like that of language can only arise from the coocurence of several states and processes, which become effects and factors, chained and potentiated to each other, then generating cascading and converging effects, developed and acting on several levels-through some aspects competing, through others collaborating.

Therefore, from its inception, language must have been the incipient result of the complex manifestation of several factors, having several causes and acting under the empire of several pressures. As language continued to be practiced, the development it went through was sequential, the various results (including those from the category of causes and factors) generating new causes, factors and effects, always engaged and generating new ways of existence and patterns of action, always becoming in a coevolutive spiral. It is quite natural, therefore, that in the different moments of its formation, functioning, spread, development, consolidation and becoming, one or the other of the observed aspects has had a weight or importance in accordance with the requirements of the stage taken. The result from the different moments, however, could only be generated by the whole complex of causes and factors, under the entire complex of pressures. As things became more and more complex, factors and causes matured, were born and-at the same time, although to different degrees-stimulated, modulated and nourished the development of complex behavior and byproduct of a biosocial nature.

Prerequisites for the emergence of human language. Under the influence of needs, organisms develop the ability to consume energy of adequate quantity and quality, in order to centralise processes that produce the adequate ways (being they initial) to address those needs. The development of a modality and of an instrument that serve social communication belongs to this category, and the means of solving them, as well as the concrete results, inextricably combine biological factors with the social ones, generating and developing slowly, gradually and semiconsciously a by-product of the biosocial evolution of a primate. The entire natural enterprise, given, on the one hand, by the imperious character of such a need, and on the other by the ways of obtaining the results, shows that the human language appeared through polygenesis, that is, it must have developed relatively simultaneously in several communities, isolated from

\footnotetext{
${ }^{1}$ In the following we will make a brief synthesis regarding the genesis and appearance of human language. In order not to list the broad and complex bibliography that helped us to study and understand this issue, we will only refer to one of the most riveting propagators of the importance of this matter, namely Michael Corballis. Although his presentations are based on an ample and quality bibliography, given the complexity of the matter, in relation to the concentrated character of our exposition, those shown by Corballis, in his articles (2003, 2007, 2009a, 2009b, 2010, 2013, 2014a, 2014b, 2017), are augmented with data, observations and conclusions that belong to us, also procured and substantiated by the branches of biology (anatomy, physiology, embryology, genetics, neurology), paleontology and other fields that compete to clarify the issue (psychology, sociology, history).
} 
each other, starting with the stage when the development of the human being and of the community demanded and allowed this fact. Then, the initial development could not be unhindered, continuous and successful, just as neither biology, nor society, nor the current vocal-articulated speech are the results of a first constitution that has succeeded and continued unhindered until today. On the contrary, in different spaces there must have been various developments that worked because they worked, after which they disappeared losing themselves (sometimes, perhaps, with traces), the continuity being one of process, and not of concrete result. Finally, as both the biosocial factors and needs continued to act in an imperious way, the developments have re-emerged and perhaps there have been failures and failures again, until a period when the results have begun to stabilize and generalize among the species.

Organic premises. Examining the issue on a phylogenetic scale leads to the conclusion that the initial roles and functions of the organs that came to compose the phonatory apparatus were related to vital processes fundamental and necessary for survival (nutrition, breathing, perception, etc.), and that the evolution under certain conditions and under certain pressures of a certain branch of hominids included various changes (behavioral, neural, structural, genetic) that led to exaptations.

Exaptation is a process of reorientation-not exclusive-of the role of a constituent part, in the direction of fulfilling another function than the initial one, possibly together with which that structure was developed. Exaptation refers to the situation in which the body adds new functions to the same structure-possibly operating small adaptive restructurings. It differs from adaptation, which refers to the situation in which, responding with its capabilities to adaptive needs (internal and/or external), the body creates adapted means for survival under certain conditions. Such a process happens slowly, without abandoning the initial roles and functions, with partial results and with the relative concordance of structural and functional states. The results of a stage are gradually reached, at the cost of difficult efforts, imposed by needs that arise later and which can delay anatomo-physiological changes accordingly. Looking at the anatomical-physiological articulatory or neural structures involved in the communication process, it is found that: a) their initial "destination" had nothing to do with communication; b) are so structurally-functionally stratified that they constitute the results of some processes of becoming; $c$ ) are so complex that their evolution must have happened gradually, over a period of time that goes beyond the origins of modern man (maximum 2 million years).

Therefore, the interrelated exercise of those elements, their coevolution, together with the resulting functional changes, could lead to the conjugation of those components and to the adaptation of the resulting complex, in a direction that would allow a certain behavior and a certain use of them (communication through vocal-articulated sounds).

Bipedalism. During the production of evolutionary acquisitions, at the anatomical level there are relatively often morpho-structural changes that are difficult to explain regarding the causes that have primed and determined their production, but which, at the same time, have the potential to bring with it a cascade of chained and mutually potentiated events-by themselves and by their consequences. Such is the raising of hominines on the hind limbs, which probably manifested itself from sporadic to frequent, and which, since it offered benefits, could be selected evolutionarily. In the case of Homo sapiens-at the ontogenetic level and from a certain age-bipedalism became permanent (or just became dominant) and became the premise of several changes. As a result, skeletal, cerebral, circulatory, respiratory and digestive changes, adaptations and developments occurred, affecting practically all organs, apparatuses and functions of the body, as well as the relationships between them. The entire causal chaining has generated various related consequences, of which, here, three are of particular interest: a) the increase in the volume of the skull and of the brain; b) lowering the larynx; and c) release of the forelimbs.

Increased cranial and brain volume. The installation of bipedalism generated adaptive-evolutionary changes in the ontogenetic patterns of the skull, which had the effect of morphological reorganization and diver- 
sification of the skull. The fact happened on three major paths: a) change in the prenatal development of the cranial base and face; b) increasing the growth rate of the skull, in the first period after birth; c) specific modification of face development - all three reflecting adaptation to bipedalism, the path to a large brain and dietary adaptations.

Being the brain the organ par excellence computationally, through it acquires the body the ability to sense similarities and differences, to classify, to compose and break down information, to conclude andon these bases - to put into action the organs. The increase in its size is due to environmental factors and diet, social factors, changes and development of sensory channels, more competitive senses and increased abilities needing a more efficient "manager", which—exceeded being a certain evolutionary thresholdimplies a larger organ.

Although the process of increasing the size of the brain accompanies the emergence and evolution of modern mammals, the fact is not necessarily associated with increasing intelligence or intellectual capacity. The increase in complexity of the brain objectively involves changes in volume, but necessarily includes with maximum necessity structural changes, with functional consequences. In this respect, the number and nature of synapses are more important than the number and nature of neurons.

In a coevolutive relationship with the brain, vocal communication-either in mammals or birds-requires a brain capable of determining and processing species-specific vocalizations. The ability to speak, therefore, was attended by morphological causes at the level of the base of the skull (the compartmentalization of the components, relative positions, volume, their relationships), however, it was not the ability to communicate that demanded a larger brain, but the growth of the brain allowed the development of the ability to communicate, only later it was possible to develop mutually-stimulating developments.

Lowering the larynx. The establishment of the phonatory apparatus results from structural developments of some components-such as the larynx, mandible or tongue-which have undergone adaptations, exaptations and functional developments that have subsequently acquired roles in vocalization.

Both from a structural and functional point of view, the larynx presents traces of successive changes adapted to the environment and to the living conditions, the coevolution between morphology and activity leading to morphological adaptations that have developed their function which, in its turn, encouraged the evolution of the structure in the sense of exaptation. In this way, the larynx-the sphincter that protected the lungs of some of the aquatic creatures-became an organ capable of allowing a high intake of air, to a certain living thing becoming an organ of speech. Although the descent of the larynx is not an exclusively human trait-it is also found in other mammals and primates-in the case of the adult human being-who presents the larynx lowered in the neck-it is the effect of an adaptive response to requests unrelated to the vocalization itself, but which he took advantage of.

Partially occurring during the evolution of the hominid, the positional changes of the larynx involved functions such as breathing, swallowing, locomotion and vocalization, as well as some evolutionary changes of these mechanisms, along with changing the relationship between phonation and articulation in order to vocalize. The process has two stages: a) descends the laryngal skeleton from the hyoid-phylogenetic, to the ancestors of humans and chimpanzees; onotogenetic in children-and b) lowers the hyoid to the mandible and the cranial base in humans.

The positioning of the larynx and pharynx in the first hominids was in the upper part of the neck, as in the current primates (excluding man). Such a position allowed breathing and a good settlement of the epiglotte, necessary for food intake, without endangering the airways, but forbade the ability to modify laryngal sounds. At birth, the human being has a part of the equipment so positioned that it is unfit for the production of speech. The fact is not due to any kind of economy (as is the case with airless lungs, for example), but shows that speech is a recent acquisition-which has not yet gone from ontogenesis to 
phylogeny. An acquisition unnecessary for the simple survival of life, a potential that can be updated not only by simple exercise-like many others - but after prior structural and morphological changes.

Release of the forelimbs. It was seen that the transition to the bipedal position was a process that happened slowly, probably starting from its accidental and sporadic occurrence, going through its adoption in certain situations for which it was appropriate and efficient, and gradually reaching its full assumption, with the loss of the adaptations of its own quadrupedal position. All the while, the mere production and adoption of the position brought with it infinitely more than its raw and direct benefits. Thus, by releasing the forelimbs, bipedalism opened the way for their use for purposes other than support and locomotion, and by changing the position of the body offered the possibility of fixing with the gaze the anterior part of the other's body (implicitly of the face). Both results paved the way for the capitalization and potentiation of body movements in general and oro-facial movements in particular. The fact that the new uses could be profitable-both by broadening the spectrum of possible actions and by obtaining new attributes of the uses themselves - could create the premise of the conjugation between the instinctive adoption and the willful or controlled adoption of the bipedal position. In this way, the sequence of operations generated their amplification, going the way from diversification to refinement.

The cascade of effects, generated by the installation of bipedalism and the exaptations thus produced, is particularly suggestive if it is noticed that, starting from the use of hands, in order to carry out some actions for which other parts of the body were used (adaptation), it is passed through the operation of new actions, until then impossible or undone (exaptation) and it leads to operations executed at unprecedented quality levels (improvement). Therefore, as a consequence of a phenomenon followed by morphological and structural changes, and by functionalizations accordingly, then as a result of the coevolution between structure, function, use and stresses, from a structure that - presumably_served swimming, then support and land movement, grabbing, defending and attacking, it comes to one that can do all these things, and many others (calligraphic writing, painting, singing musical instruments, various artistic or technical operations of infinite finesse), all at a level of accuracy and delicacy continuously perfected.

Gesture. Various anatomical and functional body determinations show that the production of gestures constitutes a natural given-more than that of sounds-which serves complex and important processes for survival. Thus, among other things, gestures are indispensable to the process of learning various actions (including that of making tools).

It is significant and relevant that for an inexperienced learner, oral explanation is difficult to understand (or rather incomprehensible), while observing the action is edifying. It is only after the action of the gesture that the production of tools can become effective. Subsequently, under combined guidance, the gesture and the word will collaborate, develop and potentiate the entire complex of activities, through the network they form in this way, possibly coevoluting. This shows the temporal prevalence of gestural communication over oral communication and means that the manufacturing (tool) activity is closely related to that of communication.

As will be seen below, primates have an active neural circuit both when (intentional) movements are produced and perceived, being able to intentionally use the joint flexibility of the limbs. This system has allowed them to achieve success in learning and transmitting gestural communication. Communication, therefore, is based on the symptom and the natural gesture-natural acts, but also capable of acquiring relevance and meaning - which shows its natural bases and shows that language-which is not reduced to articulated speech (and, implicitly, does not arise from vocalized calls) - begins with postutile and primate gestures and continues with its calls and vocalizations. By the way it appears and develops, as well as by the gradual amplification of its effects, the gesture is important both phylogenetically and ontogenetically, 
having social implications and consequences, generally participating in the cumulative cultural evolution and contributing to its development.

Even so, although oro-facial gestures-with the complement represented by the brachial ones-can communicate-and, on this basis, serve communication - they are limiting because they fulfill their role only if the individuals are quite close spatially and keep a quality visual contact. As long as the community is not too large (a few dozen individuals), the relationship between its communication needs and capacities can be harmonious, such a solution working. The increase in the size of the groups and in the number of interactions within large groups makes the gesture-which is not as noticeable, as nuanced and liberating as speech-no longer satisfy those needs at a satisfactory level. In addition, the gestural exercise leads to the loss of the iconic value of the mimicked gestures and to their conventionalization, which opens the way to the transition to the symbolism of the linguistic sign.

This form of miniaturization, done by the transition from gestural to vocal language, is a common feature for animals and stems from the natural tendency towards energy saving, being favored by the efficient and useful character of the new way, given that thus the phonatory apparatus takes over the burden of communication, by releasing hands for other activities.

However, since, in primates, the manual and facial control is superior to the vocal one, the basic elements of phonology (distinctive features, segmental elements, syllables) do not appear as a result of the spoken nature of the language and are not due exclusively to the articulatory movements. They are due to facial and manual movements. This means that vocal language developed from a system that controlled the movements of the hand and face. Speech, that is, is not a system of sound production, but one of producing articulatory movements, to which the independent action of the articulatory organs that constitute the phonatory apparatus competes, i.e. the lips, the palatine veil, the larynx and the parts of the tongue (its root, body and edges). Speech itself is a system of gestures of the tongue, lips and larynx-which is able, once again, to realize that the whole process is one of passage from one way to another and a form of manifestation of evolution.

The fact that the movements of the hand and of the phonatory apparatus are neurally, phylogenetically related, as well as under the aspects of development and behavior, shows that the gestures of the phonatory apparatus were incorporated in the primordial gestural concert, the visible gestures keeping their informational character, the vocalizations highlighting the gestural content. The possibility of using the gesture as an articulatory movement that facilitated the emergence and adoption of "speech", which responded to the growth: a) the community, b) the frequency and intensity of contacts within it, c) the need for precision in communication. The consequence of the overlapping of these movements is the coarticulation, which makes possible two results: the high rate of production and perception of sound flows.

It is thus noticed that the transition from gestural (visual) to vocal (acoustic) language knows degrees. The symptomatic character of the gestures makes them implicit, then makes them suitable for use in interindividual communication (from a producer to a certain receiver), which places them at the base of the articulating act, the course starting from the domination of the gesture that is only accompanied by the voice, then taking place the reversal of the roles, but keeping the combination of the two (still present, strong and even inextricable). Of course, the association of speech to the communication system until then has amplified the communication force and diversified the possibilities of the latter-with the increase in the need to master the system well. This process happened naturally, as a consequence of an evolution, probably determined by the face in which the organism was constituted on an evolutionary path, as the brachio-manual, oro-facial and oro-laryngal movements are controlled by structures located in the Broca area (in the other primates $\mathrm{F} 5$ area), also here being some mechanisms that link the perception to the production of the action. 
From the foregoing, an important aspect for the present discussion is already revealed, namely the multimodal character of the brain. Some of the impulses it transmits lead-in childrenboth to hand movements and to babbling. Like the brain, communication-even in its elaborate form, vocal-articulated speech-is also multimodal (plurimodality is not foreign to other primates), such communication being supported by the development of certain regions of the brain (especially the parietal ones) and their relations with others.

Mirror neurons. Constituting a natural extension of the recognition action, the mimetic capacity is involved in the unfolding and diversification of countless processes of organismal and social development and evolution. Primates hold a neural pattern from pre-homotors areas (generated by observing the action and similar to that generated by the production of the action), which constitutes a fundamental mechanism of recognition of the action made by others. In the F5 area of the prefrontal cortex of primates (less in humans) and in the Broca area (in humans) there is the mechanism that activates mirror neurons, at the moment when visual stimuli provide perceptions of an action that, once observed, can trigger the execution of that action. Although between the two areas there is no functional identity (in other primates the F5 area coordinates the muscular movements of the upper limbs, in relation to a visual stimulus or an auditory one-audio-visual mirror neurons-while in humans, in the Broca area there is the headquarters that controls the articulatory movements, in relation to an auditory stimulus, the control of the vocalization being reduced in the other primates), here are the circuits that connect the hand to the mouth so that, when, at the same time, objects are grabbed and the mouth is opened, the two openings are proportionally equal (in both directions: the mouth opens approximately on the size of the object grabbed, and the hand opens approximately on the opening of the mouth). By activating themselves both when the animal makes manual, facial and vocal movements, and when it notices others that they are doing them, the mirror neurons show the organic relationship between them.

Further, following the learned observation, not only the simple perception, but also the thought in that direction can stimulate the energetic discharge towards there, which triggers an adequate response to the observed or evoked mental behavior. Spent through mirror neurons, the process indicates the close relationship between the representation of reality and speech. Observing, then-both from the actor and the observer-that an involuntary response affects the behavior, leads to the recognition or attribution of an intention and to the establishment of a dialogue; it constitutes the core of language. Being fundamental to empathy, understanding and imitation, in the case of humans, the mechanism of mirror neurons extends to learning and language.

All these facts show that, being coupled with the mechanisms of learning, the generic structures that grant the observation of the action with its execution initiated the cultural evolution of the human languages. Going beyond and encompassing oro-facial and brachio-manual communication, the vocal-articulated language gradually appears as a result of the evolution of a basic mechanism that was originally not related to communication: the ability to recognize actions. Without constituting a human peculiarity, the gestural-manual system exploited the system of matching observation with execution and smoothed the way for the evolution of the open vocalization system, called speech.

The semi-abandonment of sign communication and the exercise in the new direction required the use of complex sound emissions (combinatorial sounds), and the anatomical possibility in this direction led the language from its brachio-manual origins to the emission of sounds. Gradually, the gestures will reduce their importance, the vocalization will gain autonomy, and the relationship will change, the gesture becoming accessory (although it does not disappear, but remains a companion who reflects the genesis of the entire functional structure-just as certain reflexes or movements remain associated with each, proving their genealogic and anatomo-physiologic relationship).

FOXP2 is a gene expressed in many regions of the brain, being present in its own and characteristic forms in the genetic endowment of many other animals - from reptiles to humans, which indicates the important role of this type of genes, in the brains of vertebrates. It has numerous roles in the development 
of the animals to which it is located, regardless of the presence or absence of any relationship with imitative voice learning.

In humans, the changes in the different regions of the brain-connected by the circuits of the basal ganglia, in relation to the FOXP2 gene-together with the mutation of this gene (very likely subject to evolutionary pressures and being the target of natural selection) favored the appearance of speech (without abandoning gestures). As indicated by several types of data, FOXP2 is involved in language, but it is by no means a "language" gene. This is not only because (with a few exceptions notable only by the spectacular effects, but not otherwise) there are no genes that hold a single and exclusive role-but also because it is involved in the development of the brain, in the formation of neural circuits with regulatory action on motor and cognitive capabilities, in the functioning of certain specific areas (with effects on language, speech and cognition), then in the development of lungs and esophagus, as well as in the control of the expression of other genes.

Through part of the complex of actions and effects that its functioning brings with it, for the present discussion, the FOXP2 gene is important because it has contributed to the coevolution of anatomical and neural adaptations related to speech and bipedalism, being deeply involved in the appearance and stabilization of speech. At the same time, it is involved in accelerating the probabilistic learning of language traits-by way of segmentation of language and action elements-aiding the transition from declarative to procedural learning, thus adapting the brain to language.

Basal ganglia. The emergence and development of basal ganglia was an important neural change for the acquisition and learning of language and language. They play an important role in higher cognitive functions-including strengthening learning and procedural memory-and emotional behavior. Although the great role of the basal ganglia refers to the motor control, since their function exceeds the motor planning and control, reaching the initiation, amplification and refinement of the signals that facilitate decision making, they are important for the control over the vocalization. In childhood, their major role consists in training the patterns of the motor vocal tract, and in adulthood, in the emotionalprosodic modulation of the statements.

The morphological changes initiated by the FOXP2 gene, at the level of the basal ganglia, provided the physiological basis necessary for the sensorimotor capabilities necessary for the articulated speech and contributed to the fine regulation of the cortical circuits of the basal ganglia. The fact is relevant for the acquisition and plasticity of language (both in humans and birds). Through the connections with the cerebellum (involved in certain cognitive functions, in the acts of articulation, of segmentation of the sound flow and of controlling the coarticulation, and which contains circuits whose role is to build and store in the long term internal representations of the world generated linguistically), the basal ganglia participate in the production and processing of language, being involved in auditory retroversion, sensorymotor adaptation and learning. Through the relations with other nervous structures of the brain and due to the circuit loops, the basal ganglia get the possibility to transmit information so that, through the developed connections, to participate in the behaviors directed towards a goal.

Regarding the process of accommodating the laryngal and supralaringal movements involved in articulation, the processing of signals related to evaluation and selection, then motor learning, the basal ganglia had a tailor-made role because, through their action, the exercise could be fixed on certain coordinates, also the results of the exercise, and the motor patterns of speech were automated. Finally, given the relationship between the basal ganglia and the rapid behavioral variation-related to voice learning - then between the prosodia subject to emotiveness and the amount of dopamine, it is seen that the basal ganglia played a major role in the new type of communication behavior.

All these facts shows the importance of basal ganglia for the process of linguistic communication, to which they contribute in many ways.

Sociality and cooperation. The biological endowments, the way of life, their practice and their adaptive evolution are able to lead to the development of the behavioral patterns of the organisms in their own 
ways. Primates are common in the ability to combine objects, to build and use tools and to perform all these actions in increasingly complex ways, the same neural circuits and areas governing the combination of symbols and the use of tools. Of course, performance levels differ, being greatly increased in the case of H. sapiens.

Recursiveness refers to the process by which a computational process appeals to itself or to a similar one, and seems to distinguish human language from the forms of communication of other animals. In reality, the fact that the elements join and merge or compose, thus forming larger structures, which, in their turn, follow the same path to generate even more extensive structures, is a universal one and knows degrees. Thus, the fractals of the inorganic world and the modularity of the organic and living world constitute species of the same process. At another level, such a process - which can be called in several ways, the reservation of a name not automatically meaning that it is thus called a special and exclusive species of reality-is a characteristic of several types of human behavior (including the cogitational one) and is not limited to language-which it precedes ${ }^{2}$. At the same time, although it manifests itself in the thinking and behaviors of the $H$. sapiens species, recursion is not a mandatory presence in natural languages, that is, it is not an indispensable condition for human language. On the other hand, although in the case of the human being it knows very advanced degrees-although not for all specimens of the speciesrecursion is not forbidden to other animals, in whose worlds it exists, therefore.

Based on the evidence obtained by the study of fossils and paleontology, it can be argued that the future H. sapiens detached from the other hominids of the family about 7-8 million years ago, its evolutionary course following stages partially similar, partly own, but essentially comparable to those of the other branches derived from the same successive trunks. Whether they continued older development directions, or they initiated new ones, those functional structures (types of organisms) had to respond, in their face, to the same environmental demands (even if otherwise felt), as well as to the internal requirements and trends. This fact could lead to the development of some particular attributes, among which the thinking and the language are emphasized, gradually acquiring their own characteristics (which is not the same as the uniqueness).

From the foregoing it is also understood that different classes (such as mammals) and, subsequently, orders of animals (such as primates) may present common structural patterns which may subsequently experience relatively common, differentiated, parallel and even divergent developments. That is, the evolution of those structures happens gradually and in continuity. Thus, for example, the neural circuit networks of primates and rodents present frontal, temporal and parietal neural systems involved in autobiographical memory, imagining the future, acquiring perspectives on others, that is, elements necessary for the existence of thought and communication. The different stage results, developments and innovations may be likely to characterize and even to customize the species, but the basis, as well as enough elements of travel are common and follow the general lines of living matter, then those of class, order, family, species, individual. Evolution is contextualized.

Based on the same types of evidence, it can be shown that about 2 million years ago, in the Pleistocene, there were quite profound environmental changes, the new conditions bringing with them new demands

\footnotetext{
${ }^{2}$ Similarly, regardless of the name, the concept that expresses the tendency of grouping (and, implicitly, of separation), somehow, of matter, refers to the action of a force that pervades both the living and the living matter (from elementary particles to human societies and thoughts). Of course, at the different levels of organization of matter, the action and effects of force are differentiated, but regardless of the complexity of the level, the forces of attraction and rejection are the instruments that lead to the cohesion of the similar or structurally identical ones, to the adhesion of the structurally non-identical, to gregarity, to (I)sociality, etc.
} 
and needs. As always, their solution came from the establishment (through reconfiguration and innovation) of new behaviors, but this fact could only take place as a consequence of the functional adaptations of the structures.

Results such as those observed by now (bipedalism and its consequences, increase in size and globularization of the skull, increase in size of the brain, complexification of the newest evolutionary acquisitionthe cortex-, various other neural changes, exaptation and refinement of the elements of the future phonatory apparatus, then the increase in the innervation of the tongue-the hypoglotal nerve being better represented in humans, compared to the large primates - the changes in the control of breathing-the thoracic region of the spine is higher in humans than in other primates, the air supply imposed by speech requiring more thoracic and abdominal muscles) were generated by the responses of biological organisms, in the context of complexification of structures and social life, the great determinant being the increase of environmental demands and the appearance of new ones. To the demands of the environment of that time, the adaptive-evolutionary response was the complexification through concatenation and modularity, and everything that followed was allowed by the development of biological equipment. The emergence and, especially, the exaptation of the mentioned structures, together with the consequences thus generated, led to their coevolution, with multiple results, such a response contributing to the control of the processes that, through gradual evolution, led to the emergence of human language.

Then, one of the effects and answers, from the social plan, was the domestication, a phenomenon that was propagated and amplified, both in itself and in effects. Thus, on the one hand, contact with a domesticated fellow man strengthens and amplifies his own domestic state, on the other hand it stimulates both the conversion of competitive situations into collaboration (for example, the common growth of children) and their harmonious combination (as in the case of real emulation). The acquisitions thus acquired tend to be used in order to establish subsequent functions, complexified and corresponding to those advanced stages. The by-products of the domestication process and the coevolution it brought about provide complex bases for the communication process (in general), including the skills of the vocal one, that is, the vocal-articulated language.

More than in the case of the other primates, due to the increasingly demanding development through exercise, of their endowments and performances, people come to distinguish themselves in terms of the relationship between the different emotions they try, then the creation and use of tools, the subsequent cortical differentiations then creating distinct, relatively modular capacities for the complex combination of objects and for grammar.

In this social framework of competitive collaboration, the vocal-articulated language comes to help all human activities, that is, it stimulates their development in certain directions. From this it can be concluded that at its appearance, language must have been part of the computational network-reasons that could explain its tendencies to dominate other behaviors. None, however, of the structures involved in language and none of their functional effects were entirely responsible for the emergence of language, which coevoluted with other socio-cultural capacities favored by biological evolution under the pressures of environmental demands, demands and internal tendencies. No mutation or leap could lead to such a complex result, which means not so much that evolutionism cannot be excluded from the equation, but that it is the only one that can provide the correct explanation of the entire complex of processes.

Mental time travel and theory of mind are two outcomes of developments that participate in the whole complex. The two phrases refer to some capacities that, again, we encounter in enough mammals and birds, but in H.sapiens, they have remarkable developments.

The research carried out in this regard could not be too extensive, profound, detailed and equal for all the investigated species. From these it follows, on the one hand, that these attributes are recorded in $H$. sapiens, but also in other animals (some mammals, especially primates, and some birds), which have episodic memory, imagine consequences for events and actions, thus behaving to determine the consequences in the prescribed sense, based on past experiences and 
observation of the environment, trying to provoke an imagined future. On the same basis it can also be stated that, in the case of other animals, the amplitude and the level of complexity recorded in H. sapiens is not encountered (due to the increased levels of recursion which the human being is capable of). In fact, the current way of communication of the human being is different from that of other animals, through grammatical and syntactic functions, through recursion and through all the attributes that transform an act into conversation.

In essence, the two refer to a complex through which, based on past experiences and the observation of the other, future possibilities and actions are estimated to be imagined and what is happening in the other's mind is noticed, thus reaching a perspective on others-also based on a complex and practiced neural network. Using the mental journey through time one can come to understanding the biography of others, to fiction and to understanding that the other may have other beliefs and beliefs than we do.

The mental time travel and the theory of mind precede language and probably contributed to its emergence. Being suitable for communicating episodes (past, present, future, planned, fictional) and sharing the advantages of the mental journey in time, language allows the communication of what is absent, through a complex system of concepts related to objects, actions, attributes, etc., carried by words, through the principles of combination, the syntax having its origin in the combination of concepts, in order to circulate episodes.

Language genesis. From all the foregoing, enough elements can be drawn to serve to outline a coherent and reliable picture of the issue under discussion.

The hierarchical and combinatorial structure of the human language evolved starting from the Pleistocene, being an adaptation to the communication needs, which has to respond to and being in relation to the increase in complexity of the social life. Such a structure has the ability to cut out the elements from which the message is constructed, which favors the emergence of a language with syntax-as a result of the incorporation of recursive structures-and requires a developed memory. At the same time, articulated speech requires radical changes at the neural level, in order to obtain control of vocalization.

The distinction between language and speech is based on the fact that the signs of language hold all the essential features of language (which initially evolved as a visual system, only gradually incorporating facial movements and vocalization (which have once been autonomous in the last 200000 years). The fact is supported by evidence of development, neurophysiology and archaeology.

Observing the situation of other primates-who have remarkable capacities to engage in the path of symbolic and nuanced communication-it is found that language is only the consequence of evolutionary developments of certain anatomo-physiologic structures, arising from the exercise oriented by special needs. The fact is shown by the anatomy and physiology of the phonatory system, by the anatomy and physiology of the brain, viewed in relation to language (tongue, larynx, etc., the Broca and Wernicke areas, and in general the constituent neural circuits and the connections between the areas involved in language). These developments, stimulated by the interaction with the environment (as it is constituted and stabilized at the level of the concrete community, i.e. in cultural frameworks) are steadfast and generate effects.

Thus, the manufacture of tools led to a tendency lateralized to the left in view of sequential movements, which proved useful to language, the lateralization consisting in the specialization towards two types of memory systems, on the left manifesting the active memory for sequential (and possibly identification) tasks, and on the right the active memory for tasks related to spatialization. This shows that lateralization is not the consequence of the specialization of the cerebral areas, but of the organization of the respective neural networks, the left hemisphere strengthening its relations with the frontal lobe, and the right one with the areas involved in the sight. 
Then, the calls of primates, starting with the involuntary ones-developed evolutionarily-depend on neural circuits located in the oldest system, namely the limbic one. In the case of $H$. sapiens, as a result of the exercise, a separate, neocortical system will develop, which will operate through the pyramidal tract and bind by synapses directly to the nuclei of the brainstem, which were responsible for the commands to the vocal chords and tongue. These changes have contributed to increased joint control and perceptual discrimination of speech sounds, essentially the dominance of speech over other modes of communication.

Therefore, the development, by organisms subjected to certain environmental demands, of sets of biological equipment, has allowed the production, practice and development of certain actions and behaviors that have been able to contribute to an overwhelming extent to the emergence of complex functions. Then, although language is partially determined by culture, speech depends on biological changes (alterations of the phonatory apparatus, characteristics of the breathing process, neural control over vocalization), which are effects of the manifestation of natural selection, also occurring as a result of the culturally contextualized functioning of some biosocial organisms. Moreover, like other complex motor capabilities, speech is not innate either, it appears evolutionarily and is gradually learned through the trial / error process.

The studies carried out in birds-which learn the production of complex vocalizations through trial and error-show that the learning process involves actions having exploratory variability and evaluation of the resulting performances, in order to acquire motor skills that lead to the improvement of the performances of this faculty. Just like in the case of humans.

All these facts strengthen the inference that the roots of a complex behavior such as language lie in the interwove and coevolution between a biological organism equipped with various possibilities of development and subjected to certain demands imposed by its amplified exercise, in an environment with stimuli acting in certain directions. It is this fact that led to the development and amplification of those possibilities that, in an increasingly demanding environment, was able to embark on a coevolutive spiral.

Given the set, complexity and especially the interwove character of many such evolutionary adaptations and exaptations, it is clear that these happened gradually, but also that the first results led to some forms of communication in the precedence of the current one, that is, in relation of proportionality with the quantity and quality resulting in the different moments, starting from a gestural language, accompanied by body and facial movements, and reaching articulated vocalizations, but without the disappearance of the foundation located at that starting point.

Just as various anatomical-physiological structures, various processes-including thought-had precursors in the animals before $H$. sapiens and did not arise after a leap-even if it is evolutionary-nor does language miraculously appear and cannot be explained by science. Although it relates to thought, language is not a form of thought (whether it arises from experience or communication, thinking is not the same as the language that expresses it and cannot be linked to any language). Having established itself as a tool for sharing experiences and knowledge, through its exercise, as well as through the knowledge it can procure (and among whose sources language is included), language has strenghtened itself as a biosocial, communication, proteic and polyvalent behavior, forcing every possible range, from superfluous to playful, from informational to aesthetic and obtaining multiple effects, from the ratchet one (through which developments and accumulations occur beyond individuals and time) to the one of dissolution factor.

All these are the results of chained evolutionary processes, at the beginnings of which even the intermediate stages were not in between. 


\section{Bibliography}

Corballis, M.C. (2003). From Hand to Mouth: The Gestural Origins of Language, în Christiansen, M.H. \& Kirby, S. (eds), Language Evolution, Oxford University Press, Oxford, p. 403-428, Crossref.

Corballis, M. C. (2007). How Language Evolved, în „Acta Psychologica Sinica”, 39 (3), p. 415-430, [online].

Corballis, M. C. (2009). Language as gesture, în „Human Movement Science”, 28 (5), p. 556-565, Crossref.

Corballis, M. C. (2009b). The evolution oflanguage, în „Annals of the New York Academy of Sciences”, 1156, p. 19-43, Crossref.

Corballis, M.C. (2010). Mirror neurons and the evolution of language, în „Brain and language”, 112 (1), p. 25-35, Crossref.

Corballis, M. C. (2013). Gestural theory of the origins of language, în Lefebvre, C., Comrie, B. \& Cohen, H. (eds), New Perspectives on the Origins of Language, John Benjamins Publishing Company, p. 171-184, Crossref.

Corballis, M. C. (2014a). Recursive Cognition as a Prelude to Language, în Lowenthal, F. \& Lefebvre, L. (eds), Language and Recursion, Springer, p. 27-36, Crossref.

Corballis, M.C. (2014b). The gradual evolution of language, în „Humana Mente: Journal of Philosophical Studies”, 7 (27), p. 39-60, [online].

Corballis, M.C. (2017). Language Evolution: A Changing Perspective, în „Trends in Cognitive Sciences”, 21 (4), p. 229-236, Crossref. 